\title{
Un modèle simple de simulation des relations microclimat - bilan carboné chez la tomate en phase végétative
}

\author{
Christian GARY \\ avec la collaboration technique de Béatrice BRUNEL \\ I.N.R.A., Station de Bioclimatologic, Centre de Recherches d'Avignon, Domaine Saint-Paul, BP9l, F84/40) \\ Montfavet
}

RÉSUMÉ

\begin{abstract}
Un modèle simple simulant la réponse au microclimat (rayonnement, température) des différents termes du bilan carboné de plantes entières de tomate en phase végétative est proposé. Il prend en compte l'existence de réserves glucidiques et leur effet tampon entre photosynthèse et croissance. Il s’appuie sur les hypothèses suivantes : (a) lactivité respiratoire est déterminée essenticllement par la quantité de substrat glucidique disponible et par la température: (b) la croissance esı limitée par la quantité d'énergie restant disponible quand l'entretien des structures existantes est assuré. Il permet en particulier une simulation correcte des évolutions de la croissance structurale pendant une période d'obscurité prolongée et de la teneur en glucides quand les activités des sources et des puits de carbone varient. Pour que le domaine de validité de ce modèle soit étendu, l'évolution de ses paramètres avec l'âge de la plante devra être déterminée.
\end{abstract}

Mots clés additionnels : Photosynthèse, réserves glucidiques, respiration, croissancé, Lycopersicon esculentum Mill.

A simple model simulating the responses to irradiance and temperature of the carbon balance of tomato plants is presented here. Sugar storage was considered as a buffer between assimilation and growth. The main hypotheses were that : (a) respiration is mainly driven by the amount of a vailable reserve carbohydrates and by temperature : (b) growth is limited by the available respiratory energy after maintenance of existing structures has been carricd out. The model allowed good simulations of structural growth during a long dark period and of carbohydrate content when carbon source and sinks activities changed. In order to extend the validity field of this model, the effect of plant age on its parameters must be determined.

Additional key words: Photosynthesis, carhohydrate reserves, respiration, growth, Lycopersicon esculentum Mill.

\section{INTRODUCTION}

Les évolutions technologiques récentes en matière de maîtrise du microclimat des serres, de mesure, de calcul et de contrôle de processus rendent possible la modulation quasi-instantanée des différents facteurs et conditions de croissance des peuplements végétaux sous serre (BAILle et al., 1987). La possibilité nouvelle d'agir avec un pas de temps réduit à quelques minutes rend indispensable une meilleure connaissance des relations instantanées entre le microclimat et les grandes fonctions du bilan carboné de la plante entière.

Ces relations ont fait l'objet de diverses tentatives de modélisation. Les courbes de réponse de la photosynthèse au rayonnement, à la teneur en $\mathrm{CO}_{2}$ ou à la température peuvent être établies expérimentalement sans difficultés. Par contre, l'action du microclimat sur les relations entre niveau des réserves glucidiques, respiration et croissance est moins accessible. Plusieurs 
modèles ont été proposés afin de rendre compte des comportements observés (RUGET, 1981). Depuis MC CREE (1970), on distingue dans la respiration une composante d'entretien, proportionnelle à la masse de matière sèche, et une composante de croissance, liée à la vitesse de croissance. En effet, la plante recycle en permanence un certain nombre de composés, avec un taux de renouvellement variable. Une part des produits de l'anabolisme est consacrée à remplacer les produits dégradés. Le maintien des gradients ioniques consomme également de l'énergie. La respiration correspondante, dite d'entretien, est par conséquent prioritaire. Son intensité est déterminée par la quantité de structures présentes et par leur qualité (PENNING DE VRIES, 1975). Elle est modulée par la température suivant une loi exponentielle (MC CREE, 1974). Le coefficient d'entretien est le coefficient de proportionalité entre masse de matière sèche et respiration d'entretien. L'énergie respiratoire supplémentaire permet la croissance. L'efficience de croissance, indépendante de la température (MC CREE, 1974), est le rapport entre la masse de matière sèche produite et la masse d'assimilats consommés (Thornley, 1970). Penning de VRies et al. (1974) calculent coefficients d'entretien et efficiences de croissance en suivant pour chaque type de constituant de la matière sèche végétale la voie métabolique qui permet sa synthèse à partir du glucose, considéré arbitrairement comme le substrat carboné de la croissance (les autres substrats possibles sont exprimés en équivalents-glucose).

La plupart des auteurs, ayant pour objectif la prévision des rendements, adoptent un pas de temps journalier. Ils négligent souvent les réserves glucidiques, considérant que les assimilats accumulés pendant la journée sont métabolisés pendant la nuit qui suit. Or MOLDAU \& Karolin (1977) ont mis en évidence sur Phaseolus vulgaris $\mathrm{L}$. un effet tampon des réserves glucidiques entre photosynthèse d'une part, et respiration et croissance d'autre part. Ce phénomène ne peut être ignoré, à plus forte raison. si le pas de temps est inférieur à la journée. Le matériel végétal utilisé est la tomate ( Lycopersicon esculentum Mill.) en phase végétative. II est susceptible d'accumuler de grandes quantités de glucides, en particulier en conditions de basses températures (Yoshioka et al., 1977). C'est pourquoi l'évolution de ce compartiment est prise en compte. Par croissance, on entend la production de matière sèche "structurale » à partir des produits immédiats de la photosynthèse. Les "structures » sont définies par opposition à ces produits de la photosynthèse et à leurs formes de transfert et de mise en réserve (Thornley, 1976). De plus, l'activité respiratoire répond à la teneur en glucides suivant une relation hyperbolique, la température agissant sur l'asymptote de cette relation (GARY, 1988). Ces données récentes permettent de discuter certaines des hypothèses retenues par GENT \& ENOCH (1983) dans le modèle de bilan carboné qu'ils proposent et qui comporte un compartiment « réserves glucidiques». C'est pourquoi nous avons repris la structure générale de leur modèle en modifiant l'expression du déterminisme de l'activité respiratoire. GENT \& ENOCH (1983) valident leur modèle sur des plantes placées en conditions climatiques stables. Notre objectif étant de simuler le comportement de plantes cultivées sous serre, nous avons voulu juger des qualités prédictives du nouveau modèle en conditions instables. Sa validation a donc été réalisée dans des conditions de rayonnement et de température variables et différentes des conditions de culture subies du semis jusqu'au moment de l'essai.

La teneur en glucides représentant à chaque instant le bilan entre l'offre (photosynthèse) et la demande (respiration, croissance) de carbone, c'est essentiellement sur ce paramètre qu'a été validé le modèle proposé ici. La croissance des structures a été estimée en moyenne sur une durée de quelques heures en réalisant un bilan carboné périodique à partir de la mesure simultanée des échanges de $\mathrm{CO}_{2}$ et des teneurs en glucides. Les essais réalisés montrent que les modifications apportées au modèle de GENT \& ENOCH (1983) permettent une bonne simulation de l'évolution des différents termes du bilan carboné.

\section{LE MODĖLE}

Considérons la plante entière comme un système qui fixe le carbone atmosphérique pour assurer sa propre croissance (fig. 1). Dans une situation où l'eau et les éléments minéraux ne sont pas limitants, 3 variables extérieures au système pilotent son fonctionnement : le rayonnement, la teneur en $\mathrm{CO}_{2}$ et la température. $\mathrm{La}$ matière sèche est divisée en un compartiment « réserves glucidiques » et un compartiment «structures » dont les contenus sont représentés par les 2 variables d'état du système. Sont désignées ici comme réserves glucidiques les formes connues de stockage transitoire (amidon) ou de transfert (glucides solubles) chez la tomate. Quatre variables de flux rendent compte des transferts de carbone entre l'atmosphère et ces 2 compartiments : la photosynthèse brute (photosynthèse nette moins respi-

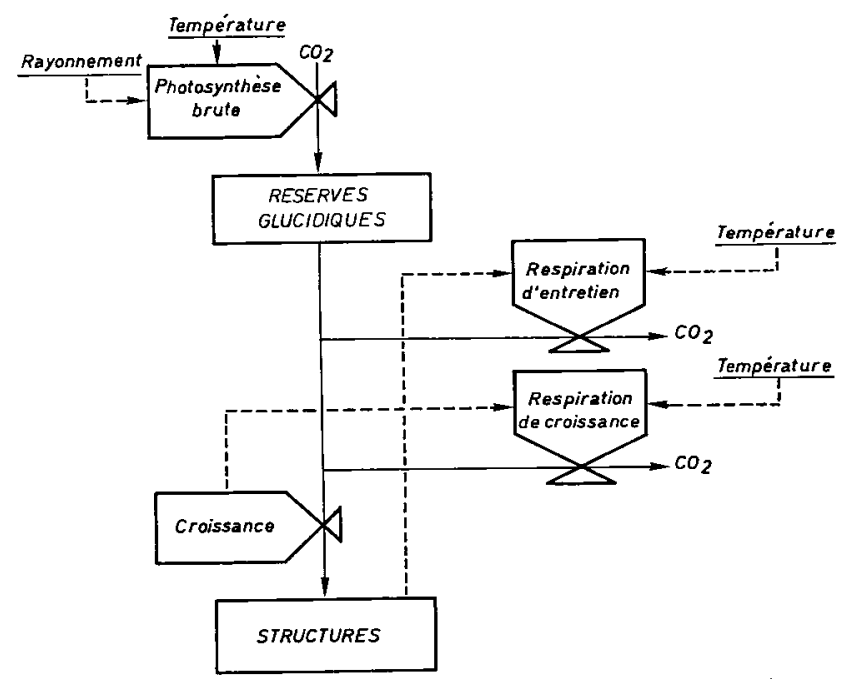

Figure 1

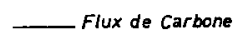

Schéma de's relations microc limat - hilan carboné, dessiné suivant les contemions decriture de ForRester 196I, cité dans PENNING DE VRIES \& VAN LAIR, 1982).

Diagram of the microclinate - carbon balance relationships, drawn according to FORRESTER (1961, mentioned by PENNING DE VRIES \& V.T L L 1 R 1982).

$\longrightarrow$ Hax de carbone/carbon flow.

-n-n flux dinformation/information flow

$\checkmark$ viriable d'état/state variable' rariable d'état/state variable
rariable de flux/flow' variable variable d'entrée/forcing variable 
ration), la respiration d'entretien, la respiration de croissance et la croissance.

Gent \& ENOCH (1983) supposent dans leur modèle de bilan carboné qu'il y a :

- un effet exponentiel de la température sur la respiration et sur la croissance ;

- une relation linéaire entre teneur en glucides et croissance ;

- indépendance des effets température et teneur en glucides sur la croissance.

GENT (1986) a, depuis, infirmé la première hypothèse en observant que la vitesse relative de croissance (RGR) présentait un maximum entre 20 et $25^{\circ} \mathrm{C}$ (sur des plants de tomate en phase végétative). Dans le cas d'une plante ayant des capacités importantes d'accumulation des glucides comme c'est le cas pour la tomate, la linéarité de la réponse de la respiration à la teneur en glucides est peu probable. Enfin, la mauvaise adéquation entre résultats expérimentaux et sorties du modèle de ces 2 auteurs dans des conditions de basses températures évoque une interaction entre les effets température et disponibilité en substrat glucidique. Ce dernier effet est d'autant moins marqué que la température est faible (GARY, 1988).

La photosynthèse brute de la plante entière, $\mathrm{P}\left(\mathrm{g} \mathrm{CO}_{2} \cdot \mathrm{m}^{-2} \cdot \mathrm{h}^{\mathrm{l}}\right)$, présente une relation hyperbolique avec le rayonnement, $\mathrm{I}\left(\mu \mathrm{mol} \cdot \mathrm{m}^{2} \cdot \mathrm{s}^{-1}\right) ; \mathrm{P}_{\max }$ est la photosynthèse maximale et $\mathrm{K}$ est une constante.

$$
P=P_{\max } \frac{I}{I+K}
$$

Le même type de relation a été observé (GARY, 1988) entre respiration totale, $\mathrm{R}\left(\mathrm{g} \mathrm{CO}_{2} \cdot \mathrm{g}^{-1} \cdot \mathrm{h}^{\mathrm{l}}\right)$, et teneur en glucides, $\mathrm{G}\left(\mathrm{g} \cdot \mathrm{g}^{-1} \mathrm{MS}\right)$ :

$$
\mathrm{R}=\mathrm{R}_{\max } \frac{\mathrm{G}}{\mathrm{G}+\mathrm{K} \frac{1}{2}}
$$

L'asymptote $R_{\max }$, dépend de la température $T\left({ }^{\circ} \mathrm{C}\right)$ suivant une loi d'Arrhenius; a et b sont des constantes.

$$
\log \left(\mathrm{R}_{\max }\right)=\mathrm{a} / \mathrm{T}+\mathrm{b}
$$

$\mathrm{K}_{\mathrm{I} / 2}$ est la teneur en glucides pour $\mathrm{R}=\mathrm{R}_{\max } / 2$. L'hypothèse est faite que la respiration mitochondriale présente la même intensité de jour que de nuit quand les paramètres qui la déterminent (température, teneur en glucides) ne changent pas (AzCón-BIETO \& OSMOND, 1983). La respiration d'entretien $\mathrm{R}_{\mathrm{e}}$ présente une réponse exponentielle à la température (MC CREE, 1974, sur trèfle et sorgho), avec $\mathrm{Q}_{10} \approx 2$, soit :

$$
\left.\left.\mathrm{R}_{\mathrm{c}}=\mathrm{K}_{\mathrm{c}} \mathrm{e}^{(0,0693(\mathrm{~T}} 25\right)\right)
$$

où $\mathrm{K}_{\mathrm{c}}$ est la respiration d'entretien à $25^{\circ} \mathrm{C}$. Soit $\mathrm{R}_{\mathrm{c}}$ la respiration de croissance :

$$
\mathrm{R}_{\mathrm{c}}=\mathrm{R}-\mathrm{R}_{\mathrm{c}}
$$

Soient RGR $\left(\mathrm{g} \cdot \mathrm{g}^{-1} \cdot \mathrm{h}^{-1}\right)$ la vitesse relative de croissance et CPF (« $\mathrm{CO}_{2}$ production factor », PENNING DE VRIES et al., 1974), la masse de $\mathrm{CO}_{2}$ dégagée pour la production d'un gramme de structures:

$$
\mathrm{RGR}=\frac{1}{\mathrm{CPF}} \cdot \mathrm{R}_{\mathrm{c}}
$$

Le coefficient PV («production value»), équivalent de l'efficience de croissance définie par THORNLEY (1970), représente la masse de structures produites par gramme de substrat glucidique consommé. CPF et PV ont été obtenus théoriquement à partir de la stechiométrie des réactions entrant en jeu dans la synthèse de structures à partir des réserves glucidiques (PENNING DE VRIES et al., 1974). Ils ont été établis pour 6 grandes catégories de composés (glucides structuraux, protéines, lipides, lignine, acides organiques, minéraux). Ils intègrent le coût énergétique des transferts et de l'absorption minérale. Connaissant la composition des structures produites à un moment donné par le matériel végétal étudié, on peut obtenir des valeurs moyennes de CPF et PV.

La variation de teneur en glucides peut être estimée à tout instant par l'équation suivante :

$\frac{\mathrm{dG}}{\mathrm{dt}}=0,682 \cdot(\mathrm{P} \cdot \mathrm{LAR}-\mathrm{R})-\left(\frac{1}{\mathrm{PV}}-0,682 \cdot \mathrm{CPF}\right) \cdot \mathrm{RGR}$

$P$ est multiplié par le rapport surface foliaire/matière sèche totale (LAR) pour homogénéiser les unités. Le premier terme représente les échanges de $\mathrm{CO}_{2}$ (un gramme de $\mathrm{CO}_{2}$ fixé permet la synthèse de $0,682 \mathrm{~g}$ d'équivalent-glucose) et le second la croissance des structures. Pour chaque gramme de structures produites, il faudra consommer $1 / \mathrm{PV}$ g d'équivalent-glucose dont $0,682 \times$ CPF g seront utilisés pour le métabolisme respiratoire.

\section{MATÉRIEL ET MÉTHODES}

\section{A. Matériel végétal}

L'estimation des paramètres du modèle et sa validation ont été réalisées sur des plants de tomate (variété Mélody) en phase végétative ( 5 à 6 semaines après le semis, 8 à 10 feuilles apparentes, premier bouquet floral non apparent). Les conditions de culture étaient les suivantes :

$$
\begin{aligned}
\text { jour: } & \text { durée }=10 \mathrm{~h}, \\
& \text { température de l'air et de la solution nutritive }= \\
& 20 \pm 1{ }^{\circ} \mathrm{C}, \\
& \text { rayonnement }=210 \pm 20 \mu \mathrm{mol} \cdot \mathrm{m}^{2} \cdot \mathrm{s} \cdot 1, \\
& \text { teneur en } \mathrm{CO}_{2}=400 \pm 20 \mathrm{ppm}, \\
\text { nuit : } & \text { durée }=14 \mathrm{~h}, \\
& \text { température de l'air et de la solution nutritive = } \\
& 15 \pm 1{ }^{\circ} \mathrm{C} .
\end{aligned}
$$

La composition de la solution nutritive, l'état des plantes ainsi que les méthodes de mesure des échanges de $\mathrm{CO}_{2}$ et de dosage des glucides (glucose, fructose, saccharose, amidon) ont été décrits par ailleurs (GARY, 1988).

\section{B. Estimation des paramètres du modèle}

Certains paramètres du modèle ont été tirés de données bibliographiques; d'autres ont fait l'objet d'es- 
timations expérimentales. Ainsi, la courbe de réponse de la photosynthèse brute au rayonnement a été obtenue à partir de mesures de photosynthèse nette, réalisées sur l'ensemble des parties aériennes en faisant varier le rayonnement. A chaque mesure, on a soustrait une activité respiratoire d'intensité équivalente à celle observée en moyenne de nuit sur le même matériel végétal et à la même température. Pour l'estimation des coefficients CPF et PV, la composition chimique moyenne des structures du matériel végétal a été tirée soit de mesures (de teneur en azote organique par la méthode Kjeldahl; de teneur en cendres par calcination) soit de données bibliographiques obtenues sur le même matériel végétal (CORNILLON, communication personnelle).

\section{Essais de validation}

Les résultats de 3 expérimentations différentes ont été utilisés pour la validation du modèle. A chaque fois, ces résultats ont été comparés aux sorties du modèle. Les simulations étaient conduites de la façon suivante. La première mesure de teneur en glucides était prise comme valeur initiale de G. Photosynthèse brute, respiration et croissance étaient estimées grâce aux équations (1) à (6) à partir des variables d'entrée que sont rayonnement et température. L'équation (7) permettait d'en déduire la nouvelle valeur de $\mathrm{G}$ après une durée égale au pas de temps choisi. L'itération était poursuivie sur G, sans nouveau calage à partir des données expérimentales.

1) Des mesures simultanées d'échanges de $\mathrm{CO}_{2}$ et de teneurs en glucides ont été réalisées sur les parties aériennes et souterraines pendant des périodes d'obscurité prolongée ( $48 \mathrm{~h}$ ) et à différentes températures, pour l'établissement des relations entre activité respiratoire, teneur en glucides et température (GARY, 1988). Les mesures de respiration ont été effectuées toutes les $10 \mathrm{~min}$, et les glucides ont été dosés aux temps $0,14,24$ et $41 \mathrm{~h}$. La masse de matière sèche structurale n'est pas accessible immédiatement. Pour estimer son évolution, il fallait connaitre également la matière sèche totale en fin de période d'obscurité, l'ćvolution de la répartition de la matière sèche entre parties aériennes et souterraines et leur teneur en carbone. La procédure de calcul était la suivante :

- à partir de la matière sèche finale et des teneurs en carbone, la masse de carbone à la fin de la période d'obscurité était évaluée ;

- à l'aide des mesures de respiration et donc des pertes de carbone, l'évolution de la masse totale de carbone pouvait être estimée pendant toute la période d'obscurité, en particulier aux 4 moments où étaient réalisés les dosages de glucides;

- afin de réduire la variabilité des résultats, les masses de carbone étaient toutes ramenées à la masse de carbone initiale; elles diminuaient donc régulièrement à partir de la valeur 1,0 ;

- connaissant les teneurs en glucides et les teneurs en carbone organique de la matière sèche structurale dans les parties aérienne et souterraine, ainsi que la répartition de la matière sèche entre elles, on pouvait alors estimer l'évolution relative de la masse de carbone structural, c'est-à-dire la croissance effective.
C'est la seule sortie " croissance » du modèle qui a été testée par ce moyen, afin de juger de la validité des coefficients CPF et PV retenus. En effet, dans l'expérience qui vient d'être décrite, les mesures d'activité respiratoire et de teneur en glucides ont servi à établir une partie du modèle; elles ne peuvent donc servir à sa validation.

2) Un $2^{\mathfrak{c}}$ essai a été conduit afin de tester la qualité prédictive du modèle à long terme et dans des conditions microclimatiques stables. Pour cela, une analyse de croissance a été réalisée sur les plantes en chambre de culture pendant toute leur phase végétative. Des plantes ont été prélevés par lots de 8 , séchées et pesées chaque semaine pendant 8 semaines, à partir de la levée. La vitesse relative de croissance a pu être ainsi estimée, en particulier au moment où les plantes étaient prélevées pour la validation du modèle. Etant données la stabilité des conditions climatiques en chambre de culture et la fréquence des prélèvements, l'hypothèse était faite que l'effet tampon des réserves glucidiques entre assimilation et croissance ne jouait plus, c'est-à-dire que la teneur en glucides moyenne ne variait pas d'une journée à l'autre. Ces valeurs expérimentales ont été comparées au RGR moyen évalué sur une journée type par le modèle. L'estimation du RGR était obtenue en prenant pour point de départ une teneur en glucides arbitraire et en faisant tourner le modèle jusqu'à ce que les sorties "croissance» et "teneur en glucides" ne varient plus d'un jour à l'autre. La stabilisation se produisait après quelques jours de simulation. Elle s'explique par le fait que toute variation de la teneur en glucides $\mathrm{G}$ a pour conséquence des variations dans le même sens de la respiration et de la croissance. Ce qui tend à réduire progressivement la variation de $\mathrm{G}$ (équation 7).

3) Enfin, une expérience particulière a été réalisée pour tester le modèle à nouveau à court terme et dans des conditions variables. Afin d'agir sur l'activité de la source et des puits de carbone, les plantes ont été soumises pendant $48 \mathrm{~h}$ à des traitements rayonnement et température contrastés (tabl. 1). Jusqu'au jour $\mathrm{j}-1$, elles ont subi les conditions climatiques normales. Au jour $j$, on a appliqué les niveaux de rayonnement apportant en $10 \mathrm{~h}$ les quantités d'énergie minimale $\left(0,36 \mathrm{MJ} \cdot \mathrm{m}^{-2} \cdot \mathrm{j}^{-1} \mathrm{PAR}\right)$ et maximale $(3,6$ $\mathrm{MJ} \cdot \mathrm{m}^{-2} \cdot \mathrm{j}^{-1} \mathrm{PAR}$ ) disponibles sous serre en hiver dans la région d'Avignon. Pendant la nuit $\mathrm{j}$, les températures imposées étaient celles généralement considérées comme extrêmes à cette même saison. Enfin, au jour $\mathrm{j}+1$, le niveau de rayonnement était élevé dans tous les cas. Pour les dosages de glucides, 4 prélèvements ont été effectués successivement en début et en fin des jours $\mathrm{j}$ et $\mathrm{j}+1$.

\section{RÉSULTATS}

\section{A. Estimation des paramètres du modèle}

A l'échelle de la plante entière, la réponse de la photosynthèse brute au rayonnement peut être considérée comme hyperbolique dans la gamme de rayonne- 
TABLEAU 1

Traitements appliqués pour la validation du modèle en conditions de ravonnement et de température variables. (a) en $\mu m o l \cdot m l^{2} \cdot s{ }^{1}$. (b) en ${ }^{\circ} \mathrm{C}$. Irradiance and night temperature treatments for the model validation in unstable condilions. (a) in $\mu \mathrm{mol} \cdot \mathrm{m}^{2} \cdot \mathrm{s}^{\prime}$, (b) in ${ }^{\circ} \mathrm{C}$.

\begin{tabular}{|c|c|c|c|c|c|}
\hline \multirow[b]{2}{*}{ Traitement } & \multicolumn{2}{|c|}{ Jour $\mathrm{j}$} & \multirow{2}{*}{$\begin{array}{l}\text { Nuit j } \\
\text { temp. } \\
\text { (b) }\end{array}$} & \multicolumn{2}{|c|}{ Jour $j+1$} \\
\hline & $\begin{array}{l}\text { rayonnement } \\
\text { (a) }\end{array}$ & $\begin{array}{l}\text { temp. } \\
\text { (b) }\end{array}$ & & $\begin{array}{l}\text { rayonnement } \\
\text { (a) }\end{array}$ & $\begin{array}{l}\text { temp. } \\
\text { (b) }\end{array}$ \\
\hline $\mathrm{rt}$ & 47 & 20 & 7 & 470 & 20 \\
\hline $\mathrm{rT}$ & 47 & 20 & 20 & 470 & 20 \\
\hline Rt & 470 & 20 & 7 & 470 & 20 \\
\hline RT & 470 & 20 & 20 & 470 & 20 \\
\hline
\end{tabular}

ment étudiée (fig. 2). A $20^{\circ} \mathrm{C}$, la relation observée est la suivante:

$$
\begin{aligned}
& \mathrm{P}=6,9 \frac{\mathrm{I}}{\mathrm{I}+1400} \quad\left(\text { en } \mathrm{gCO}_{2} \cdot \mathrm{m}^{-2} \cdot \mathrm{h}^{-1}\right) \\
& \mathrm{r}^{2}=0,952 .
\end{aligned}
$$

Ces résultats ont été obtenus en estimant l'activité respiratoire à $0,22 \mathrm{~g} \mathrm{CO}_{2} \cdot \mathrm{m}^{-2} \cdot \mathrm{h}^{-1}$ et en la soustrayant à chaque mesure d'assimilation nette.

Les paramètres des courbes de réponse de l'activité respiratoire à la teneur en glucides et à la température sont ceux déjà déterminés sur le même matériel végétal (GARY, 1988). $\mathrm{K}_{1 / 2}$ varie peu autour de $0,06 \mathrm{~g} \cdot \mathrm{g}^{-1}$. Pour les températures inférieures à $11^{\circ} \mathrm{C}$,

$$
\log \left(\mathrm{R}_{\max }\right)=-3101 / \mathrm{T}+11,5
$$

au-dessus de $11^{\circ} \mathrm{C}$,

$$
\log \left(R_{\max }\right)=-32540 / \mathrm{T}+115(\mathrm{~T} \text { en } \mathrm{K}) \text {. }
$$

Les mesures de respiration réalisées pendant des périodes d'obscurité prolongée ont montré que les valeurs de respiration d'entretien obtenues par GENT \& ENOCH (1983) sur le même matériel végétal pouvaient être retenues. Par conséquent :

$$
\mathrm{K}_{\mathrm{e}}=3,0 \mathrm{mg} \mathrm{CO} \mathrm{CO}^{-1} \cdot \mathrm{h}^{-1}
$$

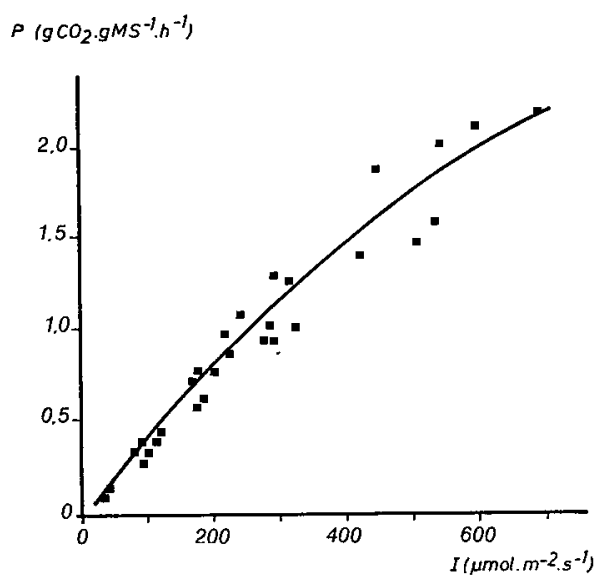

Figure 2

Courbe de réponse au rayonnement de la photosynthèse brute d'une plante entière de tomate ( 5 à 6 semaines après semis, surface foliaire moyenne $=0,06 \mathrm{~m}^{2}$ )

Gross photosynthesis of tomato plants (5 to 6 weeks after sowing, average leaf area $=0.06 \mathrm{~m}^{2}$ ) versus irradiance.
Les coefficients CPF et PV ont été calculés en considérant que la composition chimique du matériel végétal utilisé était la suivante (en $\mathrm{g} \cdot \mathrm{g}^{-1} \mathrm{MS}$ ) :

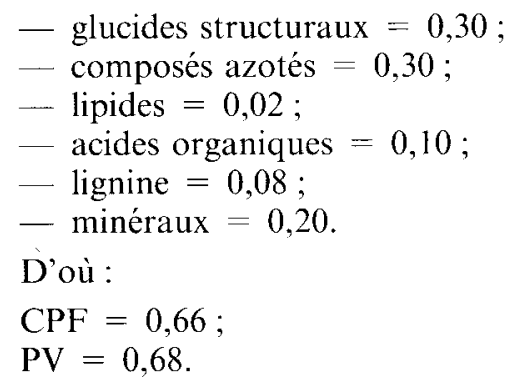

Enfin, il convenait de fixer le pas de temps du modèle, c'est-à-dire le temps pendant lequel on considère que les flux sont constants. Il doit être inférieur au cinquième de la plus petite constante de temps du système (FERRARI, 1982). La teneur en glucides est la variable d'état dont les variations sont les plus rapides: jusqu'à $0,3 \mathrm{~g}$. $\mathrm{g}^{1} \cdot \mathrm{h}^{-1}$. Le pas de temps doit donc être inférieur au cinquièrıe de $1 / 0,3 \mathrm{~h}$. Le $1 / 10^{\mathrm{c}}$ d'heure a été retenu.

\section{B. Validation du modèle}

1. Simulation de l'évolution des structures pendant une période d'obscurité prolongée

Suivant le niveau de température, l'évolution observée de la matière sèche structurale est différente (fig. $3 a$ à 3d). A $10{ }^{\circ} \mathrm{C}$, la croissance est marquée pendant les 14 premières heures, puis elle persiste à un rythme plus faible. A 15 et $20^{\circ} \mathrm{C}$, elle s'arrête au bout de 14 à $24 \mathrm{~h}$. Enfin, à $25^{\circ} \mathrm{C}$, la croissance devient négative au bout de $24 \mathrm{~h}$, ce qui laisse penser que des substrats autres que les réserves glucidiques alimentent alors la respiration. La faible croissance observée à $15^{\circ} \mathrm{C}$ serait inférieure aux croissances observées à 10 comme à $20^{\circ} \mathrm{C}$, ceci étant le résultat d'une activité respiratoire importante associée à une faible diminution de la teneur en glucides. Une sous-estimation de la teneur initiale en glucides pourrait être à l'origine de ce comportement particulier : une différence de l'ordre de 20 p. 100 sépare les 2 répétitions pour ce premier point. Rappelons qu'il n'y a pas eu d'observation directe de la croissance en matière sèche structurale mais une estimation à partir des mesures d'activité respiratoire et de teneur en glucides.

L'évolution de la matière sèche structurale donnée par le modèle est comparable à l'évolution observée (mais pas en valeur absolue à $15^{\circ} \mathrm{C}$ ). La croissance lente mais continue à $10^{\circ} \mathrm{C}$ comme la décroissance des structures 

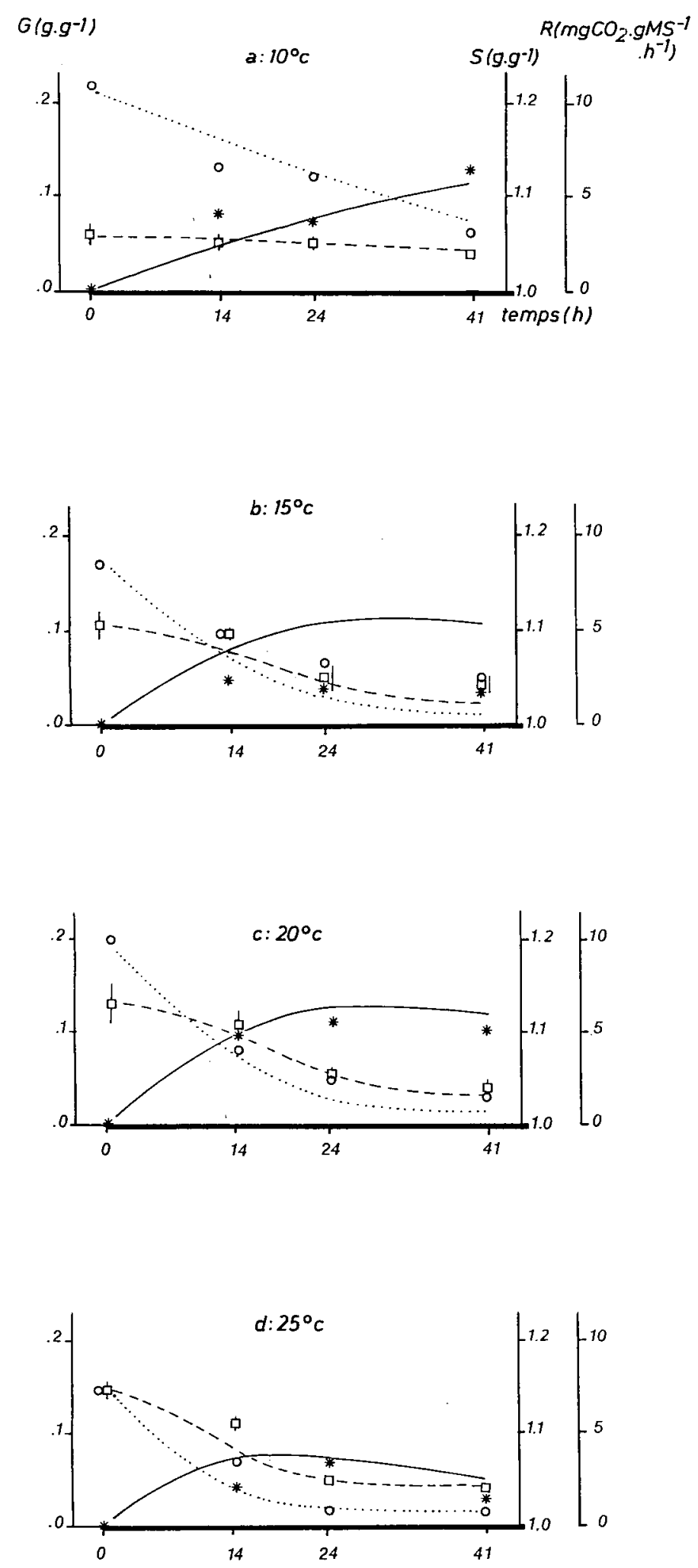

Figure 3

Evolutions mesurées (points) et simulés (traits) de la respiration $R$ $(\square,--)$, de la teneur en glucides $G(\odot, \cdots)$ ) et de la matiòre sèche structurale $S(*, \longrightarrow$ pendant une nuit prolongée do 41 heures, a différents niveaux de température. Chaque valeur expérimentale d'activité respiratoire ést la movenne des mesures pendant une heure. Il va eu 6 à 10 répétitions (intervalle de confiance $\alpha=0.10$ ). Le's teneurs en glucides sont la movenne de 2 mesur's réalisées sur des lots de 3 plantes.

Measured (dots) and simulated (lines) respiration $R(L,---)$, carbohydrate content $G(O, \cdots)$ ) and structural dry matter $S$ (*, , daring a 41 h night, at different temperatures. Everv experimental value for respiration is the average of $1 \mathrm{~h}$ measurements. There were 6 to 10 replicates (confidence interval $\alpha=0.10$ ). Experimental carbohydrate contents are the average of 2 measurements made on 3-plant 'ots. au bout de $24 \mathrm{~h}$ à $25^{\circ} \mathrm{C}$ sont bien reproduits. A $15^{\circ} \mathrm{C}$, respiration et teneurs en glucides sont systématiquement sous-estimées.

\section{Simulation de la vitesse relative de croissance moyenne sur plusieurs jours}

La vitesse relative de croissance (RGR) des plantes utilisées pour la validation a été estimée à la fin de la période de croissance en chambre climatisée (5 à 6 semaines après semis). Elle est du même ordre que celle de plantes élevées sous serre dans des conditions climatiques identiques en moyenne, mais variables d'un jour à l'autre (résultats non présentés). Le RGR moyen simulé pour la même période et dans les mêmes conditions est conforme aux résultats expérimentaux (tabl. 2). De même, la teneur en glucides prévue en début de journée est bien du même ordre que celles mesurées au début des expériences de validation qui sont présentées ci-après.

\section{TABLEAU 2}

Valeurs mesurées et simulees de la vitesse relative de croissance ( $R G R$ ) ¿t des teneurs en glucides pendant la période d'elevage des plants. Molenne = écart-type, (a) en $g \cdot g^{-1} \cdot{ }^{l}{ }^{I}$, (b) en $g \cdot g^{\prime \prime}{ }^{l}$. Le RGR est calcule a partir des masses de matière sèche moyennes de plantes entices ( $n=5$ ), mesureses aux $30^{\circ}$ et $42^{\prime}$ jours apress le semis. La teneur moleme en glucides $(n=4)$ de la plante enticre est mesuré an $42^{x^{\prime}}$ jour.

Measured and simulated $R G R$ and carbollydrate content of the secedlings. A verage $\pm S D$, (a) in $g \cdot g{ }^{\prime} \cdot d{ }^{\prime}$ ', (b) in $g \cdot g{ }^{\prime}$. RGR was calculated from averages of uhole-plant dry matter $(n=5)$ measured on the 30th and 42nd days after sowing. The average carbohydrate content $(n=4)$ of whole-plant was measured on the 42nd day.

\begin{tabular}{lccc}
\hline \hline & $\begin{array}{c}\text { RGR } \\
\text { (a) }\end{array}$ & $\begin{array}{c}\text { Teneur en glucides } \\
\text { Fin de nui } \\
\text { (b) }\end{array}$ & $\begin{array}{c}\text { Début de nuit } \\
\text { (b) }\end{array}$ \\
\hline Mesure & 0,162 & $0,065 \pm 0,012$ & - \\
Simulation & 0,156 & 0,086 & 0,195 \\
\hline
\end{tabular}

3. Simulation de l'évolution des teneurs en glucides en conditions variables de rayonnement et de température

Les hypothèses simples qui ont prévalu à l'élaboration du modèle permettent d'expliquer quelques comportements observés expérimentalement :

- l'accumulation diurne de glucides est d'autant plus importante que leur teneur est faible en début de journée (fig. $4 \mathrm{c}$ et $4 \mathrm{~d}$ ),

- la diminution nocturne de la teneur en glucides augmente avec la température, mais également avec la teneur en glucides en début de nuit (fig. $4 \mathrm{~b}$ et $4 \mathrm{~d}$ ).

Outre l'évolution des réserves glucidiques, le modèle permet de visualiser la croissance des «structures". Cette variable n'a pas été estimée dans l'expérience décrite ici, faute de mesure d'échanges de $\mathrm{CO}_{2}$. Sur le premier nycthémère, la température nocturne aurait beaucoup moins d'effet sur la croissance après une journée peu éclairée qu'après une période de forte assimilation. 

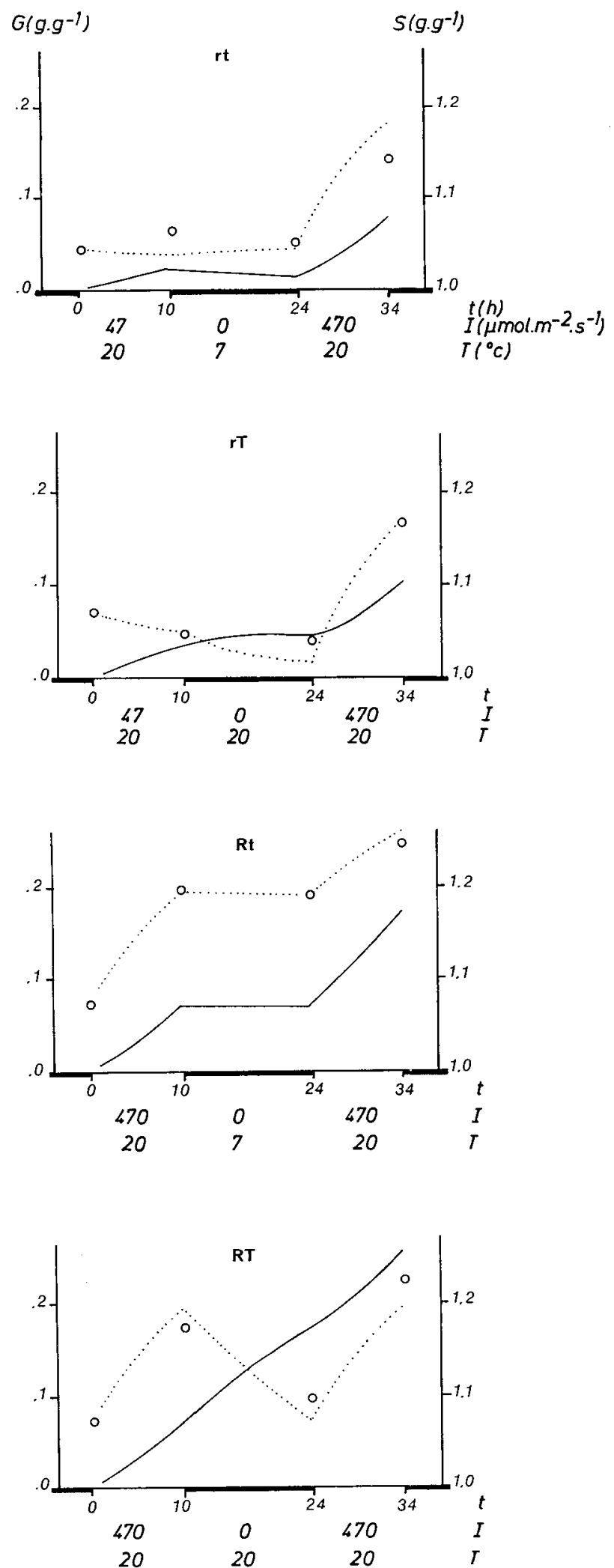

Figure 4

Evolution mesurée (points) et simulée (traits) des réserves glucidiques $G(\bigcirc, \cdots)$ et évolution simulée de la matière sèche structurale $S(\longrightarrow$ au cours de 4 séquences climatiques. Les teneurs en glucides sont la movenne de 2 mesures réalisées sur des lots de 3 plantes. Le rayonnement, I, varie au cours de la première journée (47, $470 \mu \mathrm{mol} \cdot \mathrm{m}^{2} \cdot \mathrm{s}^{\mathrm{I}}$ ) et la température, $T$, au cours de la nuit qui suit $\left(7,20^{\circ} \mathrm{C}\right)$.

Measured (dots) and simulated (lines) evolution of carbohydrate content $G(O, \cdots)$ ) and simulated structural dry matter $S(-$ during 4 climatic runs. Experimental carbohydrate contents are the average of 2 measurements made on 3-plant lots. Irradiance, I, varies during the first day $\left(47,470 \mu \mathrm{mol} \cdot \mathrm{m}^{-2} \cdot \mathrm{s}^{1}\right)$ and temperature, $T$, diring the following night $\left(7,20^{\circ} \mathrm{C}\right)$.

\section{DISCUSSION}

Ces premiers essais montrent que les hypothèses simples qui ont été retenues suffisent à simuler les relations microclimat - bilan carboné sur de jeunes plants de tomate en phase végétative. L'observation de l'évolution des structures pendant une période d'obscurité prolongée permet d'infirmer l'hypothèse de GENT \& ENOCH (1983) selon laquelle la croissance présenterait une réponse exponentielle à la température. Elle conforte plutôt l'hypothèse que nous avons retenue, selon laquelle la croissance serait limitée par la quantité d'énergie restant disponible quand l'entretien est assuré. Le dernier essai illustre l'importance de l'introduction du compartiment « réserves glucidiques » quand le pas de temps est inférieur à la journée. La prise en compte de l'interaction entre les effets température et teneur en glucides sur l'activité respiratoire permet de bien simuler l'évolution de la teneur en glucides pendant une nuit froide. L'hypothèse d'une relation linéaire et indépendante de la température entre teneur en glucides et activité respiratoire conduisait à prévoir, à tort, une diminution des réserves glucidiques pendant une nuit froide, si leur niveau était élevé en début de nuit (GENT, 1986). Le modèle du même type proposé par GENT \& ENOCH (1983) avait été validé dans des conditions climatiques stables. Celui qui est proposé ici peut fonctionner également dans des conditions variables puisque les expériences de validation ont été réalisées dans des conditions climatiques différentes des conditions de culture en chambre climatisée.

Cependant, il n'est validé que pour un type de plante particulier et sur une courte période de temps (quelques jours). En ce qui concerne le modèle photosynthèse, s'il peut rester relativement simple pour des plantes jeunes et isolées, il doit par la suite prendre en compte les effets de l'âge des feuilles sur leur activité photosynthétique et de la réduction du rayonnement disponible sous un couvert végétal qui se ferme. On ne doit pas ignorer également les phénomènes d'acclimatation :

- acclimatation physiologique : la réponse au rayonnement de la photosynthèse d'une feuille, même adulte, peut évoluer en quelques jours après que le climat lumineux ait changé (SEBbA et al., 1986);

- acclimatation morphologique : le rapport surface foliaire/matière sèche totale (LAR) est corrélé négativement avec l'assimilation nette (THORNLEY \& HURD, 1974 ; BRUGGINK \& HeUVELINK, 1987).

Sur ce dernier point, notons toutefois qu'en mesurant la matière sèche totale, la corrélation négative est en partie la conséquence de l'accumulation d'amidon, d'autant plus importante que la photosynthèse est active. Mais des modifications anatomiques ou morphologiques peuvent se produire également avec, par exemple, un épaississement des feuilles à la lumière ou un changement dans la répartition de la matière sèche entre les limbes foliaires et les autres organes. Il existe vraisemblablement un temps de latence de quelques jours entre une variation brusque de rayonnement ou de teneur en $\mathrm{CO}_{2}$ atmosphérique et une modification du LAR. Comment sont intégrées les variations journalières? II existe peu d'éléments qui permettent de prendre en compte ces phénomènes dans un modèle à pas de temps 
court comme celui qui est présenté ici. Toujours au niveau de la source du carbone, certains auteurs signalent une corrélation négative entre photosynthèse et teneur en glucides dans les feuilles (AzcoN-Bıteto, 1983). Les essais présentés ici ont été réalisés avec des niveaux de rayonnement faibles à moyens. Une éventuelle rétroaction négative de l'accumulation d'assimilats sur la photosynthèse pourrait se manifester dans des conditions de rayonnement ou de teneur en $\mathrm{CO}_{2}$ plus élevés.

On retrouve également une évolution avec l'âge de la plante au niveau de la croissance. Les coefficients CPF et PV varient avec la composition des structures produites. L'hypothèse est faite en général qu'il n'y a pas de changement à court terme de ces coefficients mais une évolution en fonction du stade de développement (PEN. NING DE VRIES \& VAN LAAR, 1982). Cela est vrai en particulier quand on passe de la phase végétative à la phase reproductrice (RUGET et al., 1981; STAHL \& MC CREE, 1988), alors qu'apparaissent des organes d'accumulation (fruits, graines...) dont le poids relatif va augmentant, et dont le métabolisme est différent de celui des organes végétatifs. La constance des coefficients CPF et PV au sein d'un nycthémère reste également à vérifier. Enfin, il convient de rappeler que les interactions entre le métabolisme carboné et les alimentations hydriques et minérales ont été exclues du champ de cette étude.

L'introduction d"un compartiment de réserves glucidiques dans un modèle classique de bilan carboné et la prise en compte des effets de la teneur en glucides et de lat température sur l'activité respiratoire permettent une bonne simulation, à court terme, de l'évolution des différents termes du bilan carboné. Le domaine de validité du modèle proposé reste réduit. Mais, sous réserve d'une meilleure connaissance de l'évolution de ses paramètres avec l'âge de la plante, il pourrait constituer un outil d'aide à la décision pour une conduite raisonnée du microclimat des serres. Il semble justifier en particulier la fixation des consignes de température nocturne en fonction du rayonnement disponible pendant la journée précédente (BAILLE et al., 1987).

Recu le 18 janvier 1988 Accepté le 31 mai 1988.

\section{RÉFÉRENCES BIBLIOGRAPHIQUES}

Azcón-Bieto J., 1983. Inhibition of photosynthesis by carbohydrates in wheat leaves. Plant Physiol., 73, 681-686.

Azcón-Bieto J., Osmond C. B., 1983. Relationship between photosynthesis and respiration. The effect of carbohydrate status on the rate of $\mathrm{CO}_{2}$ production by respiration in darkened and illuminated leaves. Plant Physiol., 71, 574-581.

Baille A., Coulon G., Jeannequin B., Wacquant C., 1987. Recent advances on use of microcomputers for greenhouse climate control and energy saving strategies in France, $5 \mathrm{p}$. In : CEC Workshop on microcomputer control systems in energy saving greenhouses, Dublin. 1-3 july 1987.

Bruggink G. T., Heuvelink E., 1987. Influence of light on the growth of young tomato, cucumber and sweet pepper plants in the greenhouse: effects on relative growth rate, net assimilation rate and leaf area ratio. Scientia Hortic., 31, 161-174.

Ferrari T. J., 1982. Introduction to dynamic simulation. p. 35-49. In F. W. T. Penning de Vries \& H. H. Van Laar, Simulation of plant growth and crop production, Sinulation monographs, PUDOC. Wageningen, $308 \mathrm{p}$.

Gary C., 1988. Relation entre température, teneur en glucides et respiration de la plante entière chez la tomate en phase végétative. Agronomie, 8 (5), 419-424.

Gent P. N., 1986. Carbohydrate level and growth of tomato plants. 2. The effect of irradiance and temperature. Plant Phisiol., 81, :0751079.

Gent P. N., Enoch H. Z., 1983. Temperature dependence of vegetative growth and dark respiration: a mathematical model. Planl Physiol., 71, 562-567

Me Cree K. J., 1970. An equation for the rate of respiration of white clover plants grown under controlled conditions, p. 221-229. In Prediction and measurement of photosinthesis productivity, Proc. IBP/PP technical meeting, Trebon, PUDOC, Wageningen.

Mc Cree K. J., 1974. Equations for the rate of dark respiration of white clover and grain sorghum, as functions of dry weight, photosynthetic rate, and temperature. Crop $S_{c i} i .14,509-514$

Moldau H., Karolin A., 1977. Effect of the reserve pool on the relationship between respiration and photosynthesis. Photosinthetica. 11 (1), 38-47.
Penning de Vries F. W. T., 1975. The cost of maintenance processes in plants. Ann. Bot., 39, 77-92

Penning de Vries F. W. T., Brunsting A. H. M., Van Laar H. H., 1974 Products, requirements and efficiency of biosynthesis : a quantitative approach. J. theor. Biol., 45, 339-377.

Penning de Vries F. W. T., Van Laar H. H., 1982. Simulation of growth processes and the model BACROS, p.114-135. In F. W. T. Penning de Vries \& H. H. Van Laar, Simulation of plam growth and crop production, Simulation nonographs, PUDOC, Wageningen, $308 \mathrm{p}$.

Ruget F., 1981. Respiration de croissance et respiration d'entretien méthodes de mesure, comparaison des résultats. Agronomie, 1 (7), $6011-610$.

Ruget F., André M., Massimino J., 1981. Evolution de la respiration et croissance, au cours d"un cycle de végetation, de maïs cultivć en chambre de mesure. Phisiol. vég., 19 (2), 277-299

Sebaa El D., Prioul J. L., Brangeon J., 1986. Acclimation of adult Lolium multiflorum leaves to changes in irradiance: effect on leaf photosynthesis and chloroplast ultrastructure. J. Plant Physiol., 127, $4.31-441$.

Stahl R. S., Mc Cree K. J., 1988. Ontogenic changes in the respiration coefficients of grain sorghum. Crop $S_{c} i, 28,111-113$.

Thornley J. H. M., 1970. Respiration, growth and maintenance in plants. Nature, 227, 304-305.

Thornley J. H. M., 1976. Mathematical models in plant physiology Academic Press, London, 318 p.

Thornley J. H. M., Hurd R. G., 1974. An analysis of the growth of young tomato plants in water culture at different light integrals and $\mathrm{CO}_{2}$ concentrations. 2. A mathematical model. Ann. Bot, 38, 389-400.

Yoshioka H., Takahashi K., Arai K., Nagaola M., 1977. Studies on the translocation and accumulation of photosynthates in fruit vegetables. 1. Effects of night- and root-temperatures as well as of the previous treatments with light intensities and nitrogen levels on the translocation and distribution of ${ }^{14} \mathrm{C}$-photosynthates in tomato plants. Bull Fe' Orn. Crops Res. Stn, A (3), 31-41 